\title{
Effets des modes de gestion des résidus du bananier et d'options de fumure minérale sur le rendement du maïs et les caractéristiques chimiques du sol dans un système de rotation bananier /maïs à l'Ouest du Burkina Faso
}

\author{
Bernard BACYE ${ }^{*}$, Hyacinthe Sami KAMBIRE ${ }^{2}$, Marie Ouindnonga SAWADOGO ${ }^{1}$ et \\ Abdoul Moumouni ZONGA ${ }^{1}$ \\ ${ }^{1}$ Université Nazi BONI (UNB), Burkina Faso. \\ ${ }^{2}$ Institut de l'Environnement et de Recherches Agricoles (INERA), Burkina Faso. \\ *Auteur correspondant ; E-mail: bbacye@gmail.com ; 01 BP 1091 Bobo-Dioulasso 01, Burkina Faso. \\ Tél : (+226) $20980635 /(+226) 70260300$
}

\begin{tabular}{lll}
\hline Received: 13-11-2020 & Accepted: 27-05-2021 & Published: 30-06-2021 \\
\hline
\end{tabular}

\section{RESUME}

Au Burkina Faso, le recyclage des résidus de culture constitue un défi pour l'amélioration de la productivité des systèmes de production agricole. L'objectif de cette étude était d'évaluer les effets de la gestion des résidus du bananier (Musa acuminata C.) et de la fumure minérale sur les caractéristiques chimiques du sol et le rendement du maïs (Zea mays L.). Un essai en split-plot avec quatre répétitions comparant quatre modes de gestion des résidus du bananier et quatre options de fumure minérale a été conduit en culture pluviale et en culture irriguée. Les rendements grains du maïs ont augmenté de 24 à 66,2\% avec le paillage et l'enfouissement de résidus par rapport au brûlage et au ramassage. Le $\mathrm{pH}$, le carbone total, l'azote total et le potassium disponible du sol ont été améliorés par le paillage, l'enfouissement et le brûlage des résidus comparativement au ramassage. L'utilisation combinée des résidus et des engrais a permis un accroissement significatif des rendements. Cependant, elle a induit une baisse du $\mathrm{pH}$ et du potassium total du sol comparativement au témoin. L'étude a montré l'intérêt du paillage et de l'enfouissement des résidus du bananier combiné avec la fumure azotée pour améliorer les rendements de maïs.

(C) 2021 International Formulae Group. All rights reserved.

Mots clés : résidus du bananier, fumure minérale, rendement du maïs, caractéristiques chimiques du sol, rotation bananier/maïs, Burkina Faso.

\section{The effects of banana tree residues managements and mineral fertilizers use options on soil chemical properties and maize yield in a banana tree / maize rotation system in western Burkina Faso}

\begin{abstract}
In Burkina Faso, the recycling of crop residues for improving the productivity of cropping systems is still a challenge. The aim of this study was to evaluate the effects of the management of banana tree (Musa acuminata C.) residues and mineral fertilizers on both soil chemical characteristics and the yield of maize (Zea mays L.). A split-plot trial with four repetitions comparing four management types of banana tree residues
\end{abstract}


(mulching, burying, burning and exporting) and four application options of mineral fertilizers was conducted in rain-fed and irrigated cropping systems. The results revealed that the maize grain yield increased by 24 to $66.2 \%$ with mulching and burying residues (green manure) compared to burning or exporting the banana tree residues. Soil $\mathrm{pH}$, total carbon and nitrogen as well as available potassium were improved by the mulching, burying and burning compared to exporting the banana tree residues. The combined use of the residues and mineral fertilizers significantly improved the maize yield. However, it caused a decrease in the soil $\mathrm{pH}$ and total potassium compared to the control treatment. The study demonstrated that mulching and burying banana tree residues in combination with mineral fertilizer improves maize yield.

(C) 2021 International Formulae Group. All rights reserved.

Key-words: banana tree residues, mineral fertilizer, maize yield, soil chemical characteristics, banana tree/maize rotation, Burkina Faso.

\section{INTRODUCTION}

L'amélioration de la productivité des systèmes agricoles constitue l'un des défis majeurs que les pays subsahariens devraient relever pour assurer la sécurité alimentaire des populations. Le recyclage des résidus organiques combiné avec des formules de fumure adaptées est considéré comme un des moyens les plus importants pour maintenir la fertilité des sols et améliorer la productivité des cultures (Djenontin et al., 2003 ; Koulibaly et al., 2010a ; Ouattara et al., 2011 ; Guto et al., 2012 et Abdou et al., 2016). Cela exige des systèmes de production, une production accrue de biomasse végétale (Vanlauwe et Giller, 2006 ; Aufray et al., 2012 ; Bacyé et al., 2019).

Au Burkina Faso, le développement de l'irrigation ces dernières années a permis d'étendre la production irriguée à des cultures comme le bananier et le maïs, en plus des cultures irriguées habituelles que sont le riz et les légumes (Barbier et al., 2011 ; Dembélé et Zangré, 2014). Selon Ariste et Kamboulé (2002), le bananier est l'une des cultures dont l'essor a bénéficié du développement de l'irrigation avec des superficies en nette augmentation. La culture du bananier présente un fort potentiel d'amélioration de la fertilité des sols (Rishirumuhirwa, 1993). En effet, la culture produit une grande quantité de résidus pouvant contribuer à l'amélioration de la fertilité des sols à travers la couverture du sol et les restitutions organiques (Roose, 2017). De plus, le bananier est une plante très exigeante en éléments fertilisants dont la culture, bénéficiant d'importantes quantités d'engrais et/ou de fumure organique (Ariste et al., 2002), pourrait avoir des arrières effets bénéfiques pour la culture suivante (Raphael et al., 2012).
Des enquêtes ont montré que dans l'Ouest du Burkina Fao, la rotation bananier/maïs occupait une part importante dans ces nouveaux agrosystèmes (Bandaogo, 2008). En effet, au moment du renouvellement des plantations de bananier après deux ou trois années de culture, le maïs vient presque toujours, soit en culture irriguée de saison sèche ou en culture pluviale. La gestion des résidus du bananier en fin de plantation devient une préoccupation pour les producteurs qui procèdent à leur brûlage suivi du ramassage des restants, afin de permettre l'installation du maïs. D'où la nécessité de trouver des options de gestion des résidus et de la fumure minérale adaptées qui permettent d'améliorer la productivité de ces nouveaux agrosystèmes.

La présente étude avait pour objectif d'évaluer, en culture pluviale et en culture irriguée, les effets à court terme de différents modes de gestion des résidus du bananier et de fumure minérale sur les rendements du maïs et les caractéristiques chimiques du sol.

\section{MATERIEL ET METHODES Site d'étude}

L'étude a été conduite dans la commune rurale de Bama à $25 \mathrm{~km}$ au Nord-Ouest de Bobo-Dioulasso au Burkina Faso. Les coordonnées géographiques sont: $11^{\circ} 22^{\prime} \mathrm{N}$, $4^{\circ} 22^{\prime} \mathrm{W}, 300 \mathrm{~m}$. Le climat est de type sudsoudanien avec une saison pluvieuse allant de mai à octobre et une saison sèche qui s'étale de novembre à avril (Guinko et Fontes, 1995). La pluviosité moyenne annuelle est de $1000 \mathrm{~mm}$.

Le sol étudié est un sol ferrugineux tropical à caractère vertique de texture argilolimoneuse avec la présence de concrétions ferrugineuses en profondeur et une profondeur 
limitée entre 60 et $100 \mathrm{~cm}$ par la cuirasse (Tableau 1). Les parcelles utilisées ont porté une culture de bananier durant trois années au cours desquelles le sol a été irrigué et a reçu des quantités importantes de fumures minérales et organiques.

\section{Matériel végétal}

La variété de maïs (Zea mays L.) utilisée (SR 21) a un rendement potentiel de 5,1 t/ha. C'est une variété à grains blancs, d'un cycle de 97 jours, résistante à la verse.

\section{Fertilisants utilisés \\ Matière organique}

Les résidus de bananier (Musa acuminata C.) constitués de feuilles et stipes ont été utilisés comme matière organique au cours de l'essai. La composition chimique de ces résidus est présentée dans le Tableau 2.

\section{Fertilisants minéraux}

Les engrais minéraux utilisés sont respectivement le NPK (15-15-15), l'urée à $46 \%$ de $\mathrm{N}$ et le superphosphate triple à $45 \%$ de $\mathrm{P}_{2} \mathrm{O}_{5}$.

\section{Dispositif expérimental}

L'essai a été réalisé selon un dispositif factoriel à quatre blocs ou répétitions avec deux facteurs étudiés. Les modes de gestion des résidus de bananier constituaient le facteur principal avec 4 niveaux: Ramassage (R); Paillage (P) ; Enfouissement (E) et Brûlage (B). La quantité de résidus utilisée dans chaque cas était de 37,5 tonnes de matière sèche par ha correspondant aux quantités estimées dans les champs après la culture du bananier. Le facteur secondaire était constitué par les options de fumure minérale avec quatre niveaux : témoin sans engrais (T_0) ; 76 kg/ha de N (T_N) soit $165 \mathrm{~kg} / \mathrm{ha} \mathrm{d}$ 'urée ; $76 \mathrm{~kg} / \mathrm{ha}$ de $\mathrm{N}$ et $30 \mathrm{~kg} / \mathrm{ha} \mathrm{de}$ $\mathrm{P}_{2} \mathrm{O}_{5}$ (T_NP) sous forme de $165 \mathrm{~kg} /$ ha d'urée et de $67 \mathrm{~kg} / \mathrm{ha}$ de superphosphate triple ; 76 $\mathrm{kg} / \mathrm{ha}$ de $\mathrm{N}+30 \mathrm{~kg} / \mathrm{ha}$ de $\mathrm{P}_{2} \mathrm{O}_{5}+30 \mathrm{~kg} / \mathrm{ha}$ de $\mathrm{K}_{2} \mathrm{O}$ (T_NPK) apportés par $200 \mathrm{~kg} / \mathrm{ha}$ d'engrais $15-15-15$ et $100 \mathrm{~kg} /$ ha d'urée. Les quantités de $\mathrm{P}_{2} \mathrm{O}_{5}$ et de $\mathrm{K}_{2} \mathrm{O}$ ont été apportées 15 jours après semis; celles de $\mathrm{N}$ ont été fractionnées à raison de $30 \mathrm{~kg} / \mathrm{ha} 15$ jours après semis et le reste 45 jours après semis. La combinaison des niveaux des facteurs a permis de constituer 16 traitements qui ont été expérimentés dans chaque bloc ou répétition avec une distribution aléatoire. Chaque traitement a occupé une parcelle élémentaire de $12 \mathrm{~m}^{2}(4 \mathrm{~m} \times 3 \mathrm{~m})$. L'essai a été conduit durant la saison pluvieuse de 2009 et la saison sèche de 2016 (campagne irriguée). L'irrigation a été faite à la raie tous les trois (3) à quatre (4) jours.

\section{Collecte des données}

Les rendements grains ont été évalués à la récolte de chacune des parcelles utiles après séchage. Les valeurs obtenues des rendements ont été extrapolées en kilogramme par hectare (kg/ha).

L'effet des traitements sur les caractéristiques chimiques du sol a été évalué sur le dispositif de 2016. Un prélèvement de l'horizon 0-20 cm de sol a été donc effectué par parcelle après la récolte. Ainsi un échantillon composite a été constitué à partir de 5 échantillons élémentaires prélevés en suivant les diagonales de chaque parcelle. Les échantillons ont été analysés au laboratoire GRN-SP de l'INERA de Farakô Bâ. Les caractéristiques chimiques suivantes ont été déterminées : le pH eau à l'aide d'un pH-mètre avec comme ratio sol-eau de 1/2,5; le carbone organique par la méthode de WALKLEY et BLACK ; l'azote total déterminé après minéralisation par la méthode KJELDAHL ; le phosphore total dosé par colorimétrie automatique après minéralisation et utilisation de molybdate d'ammonium en présence d'acide ascorbique; le phosphore assimilable déterminé par la méthode Bray I utilisant le fluorure d'ammonium $\left(\mathrm{NH}_{4} \mathrm{~F}\right)$ 0,03 $\mathrm{N}$ et l'acide chlorhydrique $\quad 0,1 \quad \mathrm{M}$ comme solution d'extraction ; le potassium total dosé après minéralisation par un spectromètre à émission de flamme ; le potassium disponible déterminé au photomètre à flamme après extraction avec une solution de $0,1 \mathrm{~N}$ de $\mathrm{HCI}$ et $4 \mathrm{~N}$ d'acide oxalique $\left(\mathrm{H}_{2} \mathrm{C}_{2} \mathrm{O} 4\right)$.

\section{Analyse statistique des données}

L'analyse statistique des données a été effectuée à l'aide du logiciel XLSTAT, version 2016. Le test de Newman Keuls a permis de comparer les moyennes au seuil de 5\% lorsque l'analyse de variance indique des différences entre les traitements. 
Tableau 1 : Caractéristiques physico-chimiques de couche $0-20 \mathrm{~cm}$ du sol ferrugineux vertique avant l'essai.

\begin{tabular}{|c|c|c|c|c|c|c|c|c|c|c|c|}
\hline \multirow[t]{2}{*}{$\begin{array}{l}\text { Profondeur } \\
(\mathrm{cm})\end{array}$} & \multicolumn{3}{|c|}{ Granulométrie } & \multicolumn{3}{|c|}{$\begin{array}{c}\text { Matière } \\
\text { organique }\end{array}$} & \multirow[t]{2}{*}{ pH eau } & \multirow[t]{2}{*}{ P total } & \multirow[t]{2}{*}{ Passi } & K total & \multirow{2}{*}{$\begin{array}{c}\mathbf{K} \\
\text { dispo } \\
\text { e sol }\end{array}$} \\
\hline & $\begin{array}{l}\text { Argile } \\
(\%)\end{array}$ & $\begin{array}{l}\text { Limon } \\
(\%)\end{array}$ & $\begin{array}{l}\text { Sable } \\
(\%)\end{array}$ & $\begin{array}{l}\mathrm{MO} \\
(\%)\end{array}$ & $\begin{array}{l}\mathrm{Ct} \\
(\%)\end{array}$ & $\begin{array}{l}\mathrm{Nt} \\
(\%)\end{array}$ & & & & mg.kg & \\
\hline $0-20$ & 29,41 & 33,34 & 37,25 & 1,45 & 1,07 & 0,07 & 5,9 & 318,0 & 40,3 & 1910,41 & 436,33 \\
\hline
\end{tabular}

Tableau 2 : Composition chimique des résidus du bananier.

\begin{tabular}{|c|c|c|c|c|c|}
\hline & $\mathrm{Ct}(\%)$ & Nt (\%) & $\mathbf{C} / \mathbf{N}$ & $P$ total $\left(\mathrm{mg} \mathrm{kg}^{-1}\right.$ de MS $)$ & $\mathrm{K}$ total $\left(\mathrm{mg} . \mathrm{kg}^{-1}\right.$ de $\left.\mathrm{MS}\right)$ \\
\hline Valeurs & 49,51 & 1,064 & 46,53 & 1277 & 27589 \\
\hline
\end{tabular}

$\mathbf{C t}=$ teneurs en carbone organique total $; \mathbf{N t}=$ teneurs en azote total $; \mathbf{P}$ total $=$ teneurs en phosphore total $; \mathbf{K}$ total $=$ teneurs en potassium total ;

MS=matière sèche.

\section{RESULTATS}

Effets des modes de gestion des résidus du bananier et des options fumure minérale sur les rendements du maïs

\section{Effets des modes de gestion des résidus du bananier}

Les résultats présentés dans le Tableau 3 montrent une différence significative $(p<0,05)$ entre les modes de gestion des résidus $\mathrm{du}$ bananier. En culture pluviale, l'enfouissement (E) et le paillage (P) ont enregistré des rendements statistiquement homogènes et significativement plus élevés que ceux du ramassage (R) et du brûlage (B) des résidus. Les augmentations de rendement varient de 843 à $1747 \mathrm{~kg} / \mathrm{ha}$. Par contre, en culture irriguée, seulement l'enfouissement a enregistré les rendements les plus élevés par rapport aux autres modes de gestion mais avec des écarts plus faibles compris entre 803 et 836 $\mathrm{kg} / \mathrm{ha}$.

\section{Effets des options de fumure minérale sur les rendements}

Les trois options apportant respectivement l'urée $\left(\mathrm{T}_{-} \mathrm{N}\right)$, l'urée et le superphosphate triple (T_NP) et l'engrais composé NPK (T_NPK) ont induit une amélioration significative $(\mathrm{p}<5 \%)$ des rendements grains par rapport au témoin sans engrais T_0 quelle que soit la saison de culture (Tableau 4). Les augmentations de rendements par rapport à T_0 varient de 1520 à 1999 kg/ha en culture pluviale et de 1631 à $2036 \mathrm{~kg} / \mathrm{ha}$ en culture irriguée. Cependant, les trois traitements n'ont pas montré de différence significative entre eux.

Effets combinés des modes de gestion des résidus du bananier et des options de fumure minérale sur les rendements

Le Tableau 5 présente les rendements grains du maïs en fonction des modes de gestion des résidus du bananier et des options de fumure minérale. Il ressort une différence significative $(\mathrm{P}<0,001)$ entre les différentes combinaisons.

Les résultats montrent que les meilleurs rendements en culture pluviale ont été obtenus avec le paillage et l'enfouissement des résidus associés à l'urée, à l'urée + le superphosphate triple ou à l'engrais composé NPK (3983 à $4917 \mathrm{~kg} / \mathrm{ha}$ contre 2619 à $3707 \mathrm{~kg} / \mathrm{ha}$ pour les autres combinaisons). Par contre, en culture irriguée, seules les combinaisons associant l'enfouissement et les fumures minérales ont enregistré les plus forts rendements (4287 à $4572 \mathrm{~kg} / \mathrm{ha}$ contre 3444 à $4142 \mathrm{~kg} / \mathrm{ha}$ pour les autres combinaisons).

Il ressort également des résultats que dans les parcelles où le brûlage (B), le paillage (P) ou l'enfouissement (E) des résidus du bananier a été pratiqué, les rendements obtenus en appliquant l'urée (N), l'urée et le 
superphosphate triple (NP) ou l'engrais composé NPK (NPK) sont statiquement identiques. Par contre, dans celles où les résidus ont été ramassés $(\mathrm{R})$, l'application $\mathrm{du}$ NPK a augmenté significativement $(p<0,001)$ les rendements grains par rapport à celle de l'urée en culture pluviale $(+1088 \mathrm{~kg} / \mathrm{ha})$. En culture irriguée, les rendements ont été également plus faibles avec l'application de l'urée mais s'ils restent statistiquement homogènes avec ceux de NP et de NPK.

Effets des différents modes de gestion des résidus du bananier et des options fumure minérale sur les caractéristiques chimiques du sol

Effets des différents modes de gestion des résidus du bananier sur les caractéristiques chimiques du sol

Dans le Tableau 6 sont comparées les caractéristiques chimiques du sol en fonction des différents modes de gestion des résidus du bananier. Il ressort que les différents modes de gestion des résidus du bananier ont affecté significativement $(\mathrm{p}<5 \%)$ le $\mathrm{pH}$ eau, les teneurs en carbone organique, en azote total et celles en $\mathrm{K}$ disponible du sol. En effet, le pH eau du sol a augmenté de 0,32 à 0,39 unités avec le paillage, l'enfouissement et le brûlage par rapport au ramassage des résidus. Les teneurs en matière organique et en azote total du sol ont été également améliorées par le paillage et l'enfouissement dont les valeurs ont augmenté respectivement de $+0,22 \%$ et $+0,02 \%$ par rapport ramassage des résidus. Concernant le potassium disponible, le brûlage se distingue nettement avec des augmentations (+47,56 à $+87,8$ mg.kg- ${ }^{1}$ de sol) par rapport aux autres de gestion. Par contre, les teneurs en phosphore total, en phosphore assimilable et en potassium total, n'ont pas montré de différence entre les modes de gestion des résidus de bananier.

Effets des options de fumure minérale sur les caractéristiques chimiques du sol

Les résultats montrent que seules les teneurs en potassium total ont été significativement affectées par les options de fumure minérale (Tableau 7). Le témoin sans engrais ( $\mathrm{T} \_0$ ) et l'apport de NPK (T_NPK), ont montré des valeurs statiquement identiques $\left(961,46\right.$ et $959,0 \quad \mathrm{mg} \cdot \mathrm{kg}^{-1}$ de sol respectivement) qui sont 1,6 fois supérieures à celles de T_NP et 2 fois à celles de T_N. Les autres paramètres chimiques analysés n'ont pas montré de différence significative entre les différentes options de fumure minérale.

Effets des modes de gestion des résidus du bananier et de fumure minérale sur les caractéristiques chimiques du sol

Les caractéristiques chimiques qui ont été significativement $(\mathrm{p}<5 \%)$ affectées par les effets des modes de gestion de résidus du bananier et des options de fumure minérale sont le $\mathrm{pH}$ eau et les teneurs en potassium total (Tableau 8). Concernant le pH eau, l'apport d'engrais (T_N, T_NP et T_NPK) a induit une diminution significative $(\mathrm{p}<5 \%)$ des valeurs de 0,18 à 0,38 unité par rapport au témoin sans engrais (T_0) avec l'enfouissement et le brûlage des résidus. Dans le cas du paillage des résidus, la valeur du $\mathrm{pH}$ eau est également élevée dans le témoin ( $\mathrm{pH} 5,42)$ mais reste statistiquement identique à celles des autres options de fumure minérale. Avec le ramassage des résidus, le témoin sans engrais (T_0) et l'apport de l'urée ( $\left.\mathrm{T} \_\mathrm{N}\right)$ sont statiquement homogènes $(\mathrm{pH}$ de 5,04 et 5,08 respectivement) et supérieurs à T_NP et T_NPK (pH de 4,99 et 4,97 respectivement).

Quant aux teneurs en potassium total, quel que soit le mode de gestion des résidus de bananier, le témoin sans engrais ( $T_{-} \_$) et l'apport de NPK (T_NPK) ont des valeurs statiquement identiques et nettement plus élevées que celles de l'option apportant l'urée $\left(\mathrm{T} \_\mathrm{N}\right)$. L'apport de l'urée et du superphosphate triple (T_NP) occupe une position médiane avec des valeurs plus faibles et statistiquement identiques lorsque les résidus sont ramassés, paillés ou enfouis $\left(640,84\right.$ à 764,07 mg. $\mathrm{kg}^{-1} \mathrm{de}$ sol) par rapport au mode brûlage $(968,56$ mg. $\mathrm{kg}^{-1}$ de sol). 
Tableau 3 : Rendement du maïs en fonction des modes gestion des résidus du bananier.

\begin{tabular}{lcc}
\hline \multirow{2}{*}{ Mode de gestion des résidus } & \multicolumn{2}{c}{ Rendement grains (kg/ha) } \\
\cline { 2 - 3 } & Culture pluviale & Culture irriguée \\
\hline Ramassage & $2642 \mathrm{a}$ & $3314 \mathrm{a}$ \\
Brûlage & $3121 \mathrm{a}$ & $3345 \mathrm{a}$ \\
Enfouissement & $3964 \mathrm{~b}$ & $4150 \mathrm{~b}$ \\
Paillage & $4389 \mathrm{~b}$ & $3347 \mathrm{a}$ \\
\hline Moyenne & 3529 & 3489 \\
\hline LSD & 799,5 & 703,5 \\
\hline CV (\%) & 27,5 & 28,5 \\
\hline Probabilité & $<0,001$ & 0,025 \\
\hline Significativité & $T H S$ & $S$
\end{tabular}

Les moyennes affectées d'une même lettre dans une même colonne ne sont pas significativement différentes au seuil de 5\% selon le test de Newman-Keuls. THS=très hautement significatif ; $\mathbf{S}=$ significatif.

Tableau 4 : Rendement du maïs en fonction des traitements de fumure minérale.

\begin{tabular}{lcc}
\hline \multirow{2}{*}{ Traitements de fumure minérale } & \multicolumn{2}{c}{ Rendement grains (kg/ha) } \\
\cline { 2 - 3 } T_0 & Culture pluviale & Culture irriguée \\
\hline T_N & $2191 \mathrm{a}$ & $2119 \mathrm{a}$ \\
T_NP & $3711 \mathrm{~b}$ & $3952 \mathrm{~b}$ \\
T_NPK & $4025 \mathrm{~b}$ & $4135 \mathrm{~b}$ \\
\hline Moyenne & $4190 \mathrm{~b}$ & $3750 \mathrm{~b}$ \\
\hline LSD & 3529,00 & 3489 \\
\hline CV (\%) & 723,60 & 483,70 \\
\hline Probabilité & 24,90 & 19,60 \\
\hline Signification & $<0,001$ & $<0,001$ \\
\hline
\end{tabular}

T_0 = témoin sans engrais; $\mathbf{T} \_\mathbf{N}=$ apport de $165 \mathrm{~kg} / \mathrm{ha}$ d'urée ; T_NP $=$ apport $165 \mathrm{~kg} / \mathrm{ha}$ d'urée et de $67 \mathrm{~kg} / \mathrm{ha}$ de superphosphate triple; $\mathbf{T}_{-}$NPK = apport $200 \mathrm{~kg} / \mathrm{ha}$ de NPK et de $100 \mathrm{~kg} /$ ha d'urée. Les moyennes affectées d'une même lettre dans une même colonne ne sont pas significativement différentes au seuil de 5\% selon le test de Newman-Keuls. THS=très hautement significatif. 
Tableau 5 : Rendement du maïs en fonction des combinaisons modes de gestion des résidus et traitements de fumure minérale.

\begin{tabular}{|c|c|c|c|}
\hline \multirow{2}{*}{$\begin{array}{l}\text { Modes de gestion } \\
\text { des résidus }\end{array}$} & \multirow{2}{*}{$\begin{array}{l}\text { Traitements de } \\
\text { fumure minérale }\end{array}$} & \multicolumn{2}{|c|}{ Rendement grains (Kg/ha) } \\
\hline & & Culture pluviale & Culture irriguée \\
\hline \multirow{4}{*}{ Brûlage } & T_0 & $2037 \mathrm{~b}$ & 1929 a \\
\hline & $\mathbf{T} \_\mathbf{N}$ & $3631 \mathrm{~d}$ & $3758 \mathrm{bcd}$ \\
\hline & T_NP & $3431 \mathrm{~cd}$ & 3939 cde \\
\hline & T_NPK & $3385 \mathrm{~cd}$ & $3754 \mathrm{bcd}$ \\
\hline \multirow{4}{*}{ Enfouissement } & T_0 & $2589 \mathrm{bc}$ & $3069 \mathrm{~b}$ \\
\hline & $\mathbf{T} \_\mathbf{N}$ & 3983 def & $4572 \mathrm{de}$ \\
\hline & T_NP & 4534 efg & $4675 \mathrm{e}$ \\
\hline & T_NPK & $4749 \mathrm{fg}$ & 4287 cde \\
\hline \multirow{4}{*}{ Paillage } & T_0 & $3217 \mathrm{~cd}$ & $1759 \mathrm{a}$ \\
\hline & $\mathbf{T} \_\mathbf{N}$ & $4611 \mathrm{fg}$ & $3467 \mathrm{bc}$ \\
\hline & T_NP & $4810 \mathrm{fg}$ & $3784 \mathrm{bcd}$ \\
\hline & T_NPK & $4917 \mathrm{fg}$ & $3444 \mathrm{bc}$ \\
\hline \multirow{4}{*}{ Ramassage } & T_0 & $919 \mathrm{a}$ & $1720 \mathrm{a}$ \\
\hline & $\mathbf{T} \_\mathbf{N}$ & $2619 b c$ & $3514 \mathrm{bc}$ \\
\hline & T_NP & $3324 \mathrm{~cd}$ & 4142 cde \\
\hline & T_NPK & $3707 \mathrm{de}$ & 4012 cde \\
\hline \multicolumn{2}{|c|}{ Moyenne } & 3529,00 & 3489 \\
\hline \multicolumn{2}{|c|}{ LSD } & 877,40 & 845,10 \\
\hline \multicolumn{2}{|c|}{ CV (\%) } & 14,90 & 17,00 \\
\hline \multicolumn{2}{|c|}{ Probabilité } & $<0,001$ & $<0,001$ \\
\hline \multicolumn{2}{|c|}{ Signification } & $T H S$ & THS \\
\hline
\end{tabular}

T_0 = témoin sans engrais ; T_N = apport de $165 \mathrm{~kg} / \mathrm{ha}$ d'urée ; T_NP = apport $165 \mathrm{~kg} / \mathrm{ha}$ d'urée et de $67 \mathrm{~kg} / \mathrm{ha} \mathrm{de}$ superphosphate triple ; $\mathbf{T} \_$NPK = apport $200 \mathrm{~kg} / \mathrm{ha}$ de NPK et de $100 \mathrm{~kg} /$ ha d'urée. Les moyennes affectées d'une même lettre dans une même colonne ne sont pas significativement différentes au seuil de $5 \%$ selon le test de Newman-Keuls. THS=très hautement significatif. 


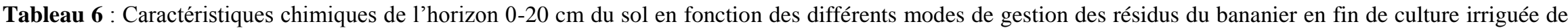
2016.

\begin{tabular}{|c|c|c|c|c|c|c|c|c|}
\hline \multirow{3}{*}{$\begin{array}{l}\text { Modes de } \\
\text { gestion des } \\
\text { résidus }\end{array}$} & \multicolumn{8}{|c|}{ Caractéristiques chimiques du sol } \\
\hline & \multirow[t]{2}{*}{ pH eau } & \multirow[t]{2}{*}{$\mathrm{C}(\%)$} & \multirow[t]{2}{*}{$\mathbf{N}(\%)$} & \multirow[t]{2}{*}{$\mathbf{C} / \mathbf{N}$} & P total & P ass & K total & K dispo \\
\hline & & & & & \multicolumn{4}{|c|}{ mg.kg-1 de sol } \\
\hline Ramassage & $5,02^{\mathbf{b}} \pm 0,18$ & $1,18^{\mathbf{b}} \pm 0,21$ & $0,09^{\mathbf{b}} \pm 0,02$ & $12,69 \pm 1,12$ & $349,00 \pm 88,00$ & $69,06 \pm 29,4$ & $769,51 \pm 249,56$ & $111,83^{\mathbf{b}} \pm 38,82$ \\
\hline Paillage & $5,34^{\mathrm{a}} \pm 0,20$ & $1,40^{\mathbf{a}} \pm 0,22$ & $0,11^{\mathrm{a}} \pm 0,02$ & $12,64 \pm 0,78$ & $402,65 \pm 101,47$ & $88,03 \pm 36,8$ & $729,04 \pm 259,93$ & $146,28^{b} \pm 55,34$ \\
\hline Enfouissement & $5,41^{\mathrm{a}} \pm 0,28$ & $1,41^{\mathrm{a}} \pm 0,23$ & $0,11^{\mathrm{a}} \pm 0,02$ & $12,75 \pm 0,63$ & $354,43 \pm 44,98$ & $63,29 \pm 18,0$ & $744,97 \pm 265,61$ & $152,07^{\mathbf{b}} \pm 54,64$ \\
\hline Brûlage & $5,35^{\mathrm{a}} \pm 0,29$ & $1,27^{\mathbf{a b}} \pm 0,23$ & $0,10^{\mathbf{a b}} \pm 0,02$ & $12,67 \pm 0,70$ & $398,08 \pm 116,92$ & $80,03 \pm 36,2$ & $934,41 \pm 284,36$ & $199,63^{a} \pm 83,90$ \\
\hline Probabilité & $<0,0001$ & $\mathbf{0 , 0 1 1}$ & $\mathbf{0 , 0 1 1}$ & 0,984 & $\mathbf{0 , 2 2 0}$ & $\mathbf{0 , 1 1 9}$ & 0,117 & 0,002 \\
\hline Signification & THS & $\mathbf{S}$ & $\mathbf{S}$ & NS & NS & NS & NS & HS \\
\hline
\end{tabular}

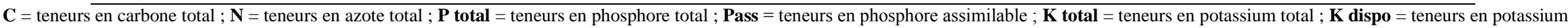

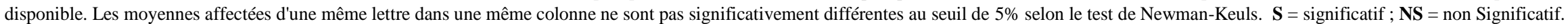

Tableau 7 : Caractéristiques chimiques du sol en fonction des options de fumure minérale.

\begin{tabular}{|c|c|c|c|c|c|c|c|c|}
\hline \multirow{3}{*}{$\begin{array}{l}\text { Options de } \\
\text { fumure } \\
\text { minérale }\end{array}$} & \multicolumn{8}{|c|}{ Caractéristiques chimiques du sol } \\
\hline & \multirow[t]{2}{*}{ pH eau } & \multirow[t]{2}{*}{$\mathrm{C}(\%)$} & \multirow[t]{2}{*}{$\mathbf{N}(\%)$} & \multirow[t]{2}{*}{$\mathbf{C} / \mathbf{N}$} & P total & P ass & K total & K dispo \\
\hline & & & & & \multicolumn{4}{|c|}{ mg.kg ${ }^{-1}$ de sol } \\
\hline T_0 & $5,42 \pm 0,35$ & $1,37 \pm 0,27$ & $0,11 \pm 0,02$ & $12,67 \pm 0,74$ & $385,50 \pm 81,02$ & $78,55 \pm 23,8$ & $961,46^{\mathbf{a}} \pm 142,82$ & $171,28 \pm 70,93$ \\
\hline $\mathbf{T} \_\mathbf{N}$ & $5,24 \pm 0,27$ & $1,29 \pm 0,22$ & $0,10 \pm 0,02$ & $13,07 \pm 0,66$ & $354,73 \pm 86,28$ & $69,78 \pm 35,7$ & $484,55^{\mathrm{c}} \pm 251,49$ & $144,45 \pm 60,88$ \\
\hline $\mathbf{T} \_\mathbf{N P}$ & $5,26 \pm 0,23$ & $1,22 \pm 0,20$ & $0,10 \pm 0,02$ & $12,65 \pm 0,87$ & $373,25 \pm 12,00$ & $73,76 \pm 36,5$ & $772,92^{b} \pm 194,86$ & $136,83 \pm 56,79$ \\
\hline T_NPK & $5,19 \pm 0,23$ & $1,37 \pm 0,25$ & $0,11 \pm 0,02$ & $12,35 \pm 0,76$ & $390,68 \pm 89,46$ & $78,32 \pm 31,9$ & $959,00^{\mathbf{a}} \pm 163,71$ & $157,26 \pm 77,95$ \\
\hline Probabilité & $\mathbf{0 , 1 2 0}$ & $\mathbf{0 , 1 9 0}$ & 0,069 & 0,092 & 0,711 & $\mathbf{0 , 8 4 9}$ & $<0,0001$ & 0,493 \\
\hline Signification & NS & NS & NS & NS & NS & NS & $\mathbf{S}$ & NS \\
\hline
\end{tabular}

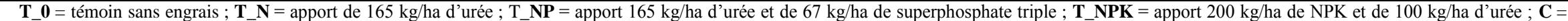

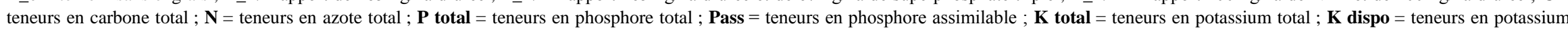

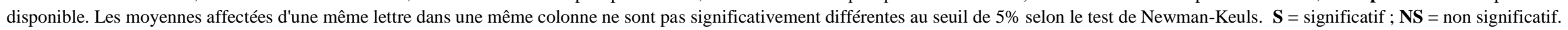


B. BACYE et al. / Int. J. Biol. Chem. Sci. 15(3): 1208-1221, 2021

Tableau 8 : Caractéristiques chimiques du sol en fonction des différents modes de gestion des résidus du bananier et des traitements de fumure minérale.

\begin{tabular}{|c|c|c|c|c|c|c|c|c|c|}
\hline \multirow[t]{2}{*}{ Modes de gestion } & \multirow[t]{2}{*}{ Traitements } & \multirow[t]{2}{*}{ pH eau } & \multirow{2}{*}{$\begin{array}{l}\text { C } \\
(\%)\end{array}$} & \multirow{2}{*}{$\begin{array}{l}\mathrm{N} \\
(\%)\end{array}$} & \multirow[t]{2}{*}{$\mathrm{C} / \mathrm{N}$} & P total & $\mathbf{P}$ ass & K total & K dispo \\
\hline & & & & & & \multicolumn{4}{|c|}{ mg.kg-1 de sol } \\
\hline \multirow{4}{*}{ Ramassage } & T_0 & $5,04^{\mathbf{a b}} \pm 0,14$ & $1,14 \pm 0,24$ & $0,091 \pm 0,02$ & $12,67 \pm 1,16$ & $341,81 \pm 79,54$ & $70,47 \pm 23,1$ & $915,16^{\mathbf{a b}} \pm 171,15$ & $102,68 \pm 18,41$ \\
\hline & $\mathbf{T} \_\mathbf{N}$ & $5,08^{\mathbf{a b}} \pm 0,29$ & $1,16 \pm 0,20$ & $0,088 \pm 0,01$ & $13,12 \pm 0,34$ & $337,29 \pm 80,72$ & $75,84 \pm 39,3$ & $601,87^{\mathbf{c d}} \pm 320,13$ & $144,15 \pm 53,34$ \\
\hline & T_NP & $4,99^{\mathbf{b}} \pm 0,18$ & $1,18 \pm 0,20$ & $0,092 \pm 0,02$ & $12,92 \pm 1,64$ & $320,93 \pm 72,10$ & $57,73 \pm 25,7$ & $640,84^{c} \pm 211,01$ & $78,29 \pm 22,31$ \\
\hline & T_NPK & $4,97^{\mathbf{b}} \pm 0,11$ & $1,22 \pm 0,26$ & $0,101 \pm 0,02$ & $12,04 \pm 1,13$ & $395,96 \pm 128,21$ & $72,20 \pm 36,5$ & $920,17^{\mathbf{a b}} \pm 130,44$ & $122,20 \pm 26,53$ \\
\hline \multirow{4}{*}{ Paillage } & T_0 & $5,42^{\mathbf{a b}} \pm 0,25$ & $1,41 \pm 0,19$ & $0,113 \pm 0,02$ & $12,46 \pm 0,49$ & $401,20 \pm 112,94$ & $88,56 \pm 26,8$ & $929,50^{\mathbf{a b}} \pm 181,62$ & $174,63 \pm 57,44$ \\
\hline & $\mathbf{T} \_\mathbf{N}$ & $5,34^{\mathbf{a b}} \pm 0,15$ & $1,38 \pm 0,24$ & $0,104 \pm 0,02$ & $13,32 \pm 0,67$ & $367,12 \pm 80,93$ & $79,30 \pm 29,8$ & $367,07^{\mathbf{d e}^{\prime}} \pm 37,09$ & $149,02 \pm 69,49$ \\
\hline & T_NP & $5,34^{\mathbf{a b}} \pm 0,25$ & $1,30 \pm 0,19$ & $0,104 \pm 0,02$ & $12,64 \pm 0,78$ & $423,80 \pm 155,19$ & $98,75 \pm 61,4$ & $719,19^{\mathbf{b c}} \pm 97,61$ & $141,71 \pm 66,57$ \\
\hline & T_NPK & $5,25^{\mathbf{a b}} \pm 0,17$ & $1,50 \pm 0,31$ & $0,123 \pm 0,02$ & $12,14 \pm 0,88$ & $418,48 \pm 73,95$ & $85,49 \pm 33,5$ & $900,42^{\mathbf{a b}} \pm 162,36$ & $119,76 \pm 26,53$ \\
\hline \multirow{4}{*}{ Enfouissement } & T_0 & $5,61^{a} \pm 0,32$ & $1,50 \pm 0,23$ & $0,116 \pm 0,01$ & $12,87 \pm 0,73$ & $382,58 \pm 18,93$ & $70,87 \pm 12,03$ & $949,80^{\mathbf{a b}^{\mathbf{b}}} \pm 75,62$ & $180,73 \pm 82,53$ \\
\hline & $\mathbf{T} \_\mathbf{N}$ & $5,33^{\mathbf{a b}} \pm 0,27$ & $1,39 \pm 0,24$ & $0,109 \pm 0,01$ & $12,70 \pm 1,02$ & $349,15 \pm 78,48$ & $53,23 \pm 17,38$ & $337,75^{\mathrm{e}} \pm 104,05$ & $117,32 \pm 17,53$ \\
\hline & T_NP & $5,43^{\mathbf{a b}} \pm 0,11$ & $1,26 \pm 0,20$ & $0,098 \pm 0,02$ & $12,86 \pm 0,41$ & $335,35 \pm 18,07$ & $59,70 \pm 17,70$ & $763,07^{\mathbf{b c}} \pm 52,82$ & $156,34 \pm 48,68$ \\
\hline & T_NPK & $5,26^{\mathbf{a b}} \pm 0,35$ & $1,49 \pm 0,22$ & $0,119 \pm 0,02$ & $12,59 \pm 0,40$ & $350,64 \pm 40,93$ & $69,39 \pm 24,28$ & $929,27^{\mathbf{a b}} \pm 103,16$ & $153,90 \pm 51,99$ \\
\hline \multirow{6}{*}{ Brûlage } & T_o & $5,60^{\mathbf{a}} \pm 0,37$ & $1,44 \pm 0,35$ & $0,113 \pm 0,02$ & $12,68 \pm 0,71$ & $416,39 \pm 96,02$ & $84,31 \pm 33,08$ & $1051,39^{\mathrm{a}} \pm 137,13$ & $227,07 \pm 64,39$ \\
\hline & $\mathbf{T} \_\mathbf{N}$ & $5,23^{\mathbf{a b}} \pm 0,34$ & $1,23 \pm 0,17$ & $0,094 \pm 0,01$ & $13,16 \pm 0,52$ & $365,35 \pm 131,14$ & $70,77 \pm 55,62$ & $631,52^{c} \pm 327,52$ & $167,32 \pm 94,04$ \\
\hline & T_NP & $5,27^{\mathbf{a b}} \pm 0,11$ & $1,13 \pm 0,22$ & $0,092 \pm 0,01$ & $12,20 \pm 0,86$ & $412,93 \pm 163,09$ & $78,85 \pm 20,77$ & $968,56^{\mathbf{a b}} \pm 233,95$ & $170,98 \pm 46,51$ \\
\hline & T_NPK & $5,29^{\mathbf{a b}} \pm 0,17$ & $1,27 \pm 0,10$ & $0,101 \pm 0,01$ & $12,65 \pm 0,56$ & $397,65 \pm 114,73$ & $86,20 \pm 41,61$ & $1086,16^{\mathrm{a}} \pm 225,08$ & $233,17 \pm 122,70$ \\
\hline & Probabilité & $\mathbf{0 , 0 0 8}$ & 0,215 & 0,114 & $\mathbf{0 , 7 3 0}$ & 0,943 & $\mathbf{0 , 9 2 0}$ & $<0,0001$ & 0,063 \\
\hline & Signification & HS & NS & NS & NS & NS & NS & THS & NS \\
\hline
\end{tabular}

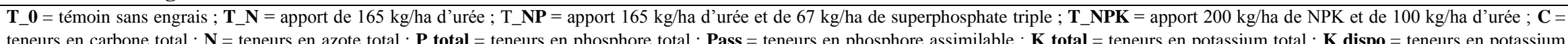

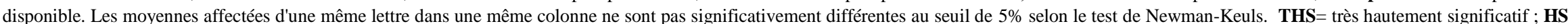
$=$ hautement significatif $; \mathbf{N S}=$ non significatif. 


\section{DISCUSSION}

\section{Effets sur les rendements grains du maïs}

Le paillage et l'enfouissement des résidus de bananier non transformés ont amélioré significativement les rendements en maïs par rapport à leur ramassage ou leur brûlage. Ces résultats confirment les effets positifs des restitutions organiques rapportés par plusieurs auteurs (Lekasi et al., 2001 ; Djenontin et al., 2003; Koulibaly et al., 2010b ; Guto et al., 2012 ; Macon et al., 2014 ; Roose, 2017). Cependant, ils ne corroborent pas ceux de Kimani et al. (2007) qui ont montré que l'apport de résidus de maïs n'a pas eu un effet significatif sur les rendements du maïs à la première saison.

Le paillage des résidus est une pratique permettant d'améliorer la disponibilité de l'eau dans le sol au cours de la saison de culture à cause de son effet positif sur la réduction du ruissellement et de l'évaporation et sur l'augmentation de l'infiltration (Cattan et al., 2006 ; Hiel et al., 2016 ; Roose, 2017). Il peut aussi contribuer à la fourniture d'éléments nutritifs à la culture à travers la minéralisation des résidus (Raphael et al., 2012). Mais selon Roose (2017), la contribution de ce processus dépend de la quantité des résidus et de leur aptitude à la décomposition. De plus, la minéralisation des résidus à la surface du sol s'accompagne de processus de pertes pouvant limiter la contribution du paillage à la nutrition minérale de la culture, surtout en ce qui concerne l'azote. Il ressort de nos résultats que l'effet à court terme du paillage a été plus significatif en culture pluviale par rapport à la culture irriguée. Ces résultats s'expliqueraient plus par l'effet du paillage sur le stockage de l'eau dans le sol dont l'impact sur les rendements des cultures n'est significatif qu'en conditions de déficit hydrique (Hiel et al., 2016; Abarchi et al., 2018) comme c'est souvent le cas dans la zone d'étude. En effet, Ouédraogo et al. (2010) et Sarr et al. (2011) ont rapporté que dans cette zone, les saisons pluvieuses sont caractérisées par une pluviométrie irrégulière occasionnant des poches de sécheresse plus ou longues avec des déficits en eau pour les cultures. Ce qui expliquerait aussi que dans les conditions de la culture irriguée où l'eau a été apportée de manière régulière au maïs le paillage ait montré un effet moindre. Pour autant, ces résultats ne signifient pas que le paillage n'ait pas d'intérêt en culture irriguée car son effet positif sur conservation de l'eau dans le sol pourrait permettre de réduire le nombre d'arrosages.

La pratique de l'enfouissement des résidus de bananier a montré un effet positif sur le rendement quelle que soit la saison de culture. Ces résultats montrent que l'enfouissement des résidus de bananier n'a pas eu un effet dépressif sur les rendements du maïs comme cela a été rapporté pour d'autres types de matières organiques (Kimani et al., 2007 ; Barthès et al., 2010 ; Barthès et al., 2015). Cela s'expliquerait par la rapide décomposition de ces résidus qui disparaissent totalement dans le sol permettant ainsi de libérer les éléments pour la culture avec une faible immobilisation (Thieureux, 2006). Selon Raphael et al. (2012), la demi-vie des résidus de bananier enfouis au champ est de 32 jours.

La gestion combinée de résidus du bananier et de la fumure minérale a permis une amélioration des rendements du maïs. Djenontin et al. (2003), Kimani et al. (2007), Badou et al. (2013) et Sohou et al. (2017) ont obtenu des résultats similaires avec d'autres types de résidus. Mais les résultats ont montré que, quel que soit le mode de gestion des résidus, l'apport d'une fumure azotée permet d'avoir des rendements en maïs grains comparables à ceux des fumures apportant l'azote et le phosphore ou les trois éléments NPK. Cela semble indiquer que dans ces agrosystèmes l'azote constitue l'élément le plus limitatif pour la production du maïs. Ce qui expliquerait les faibles rendements obtenus avec le brûlage qui ne permet pas la restitution de cet élément par rapport paillage et surtout à l'enfouissement. Cependant, lorsque les résidus sont ramassés, la fumure azotée seule paraît insuffisante pour améliorer les 
rendements du maïs; l'apport d'une fumure complète devient alors indispensable.

\section{Effets sur les caractéristiques chimiques du sol}

Le recyclage des résidus de bananier par le paillage, l'enfouissement et le brûlage, a permis un accroissement du $\mathrm{pH}$ et des teneurs en carbone total, azote total et potassium disponible dans la couche $0-20 \mathrm{~cm}$ du sol par rapport au ramassage. Ces résultats confirment le rôle néfaste de l'exportation des résidus sur l'entretien de la fertilité des sols (Koulibaly et al., 2010a). Ils corroborent ceux rapportés par Barthès et al. (2010) qui ont montré une amélioration des caractéristiques du sol, notamment du statut organique suite à l'apport de bois raméaux fragmentés. Cependant d'autres travaux ont, au contraire, montré que l'apport des résidus de culture n'avait pas un effet significatif sur les caractéristiques du sol à court terme (Barthès et al., 2010 ; Barthès et al., 2015 ; Roose, 2017). Cette absence d'effet des apports organiques se justifierait par les faibles quantités apportées et la pauvreté des résidus en éléments minéraux (Roose, 2017). Aussi nos résultats s'expliqueraient-ils par les fortes quantités de résidus de bananier apportés ainsi que la bonne aptitude à la biodégradation de ces résidus rapportée par Thieuleux (2006). L'augmentation très marquée des teneurs en potassium disponible dans le traitement brûlage s'expliquerait par l'effet de minéralisation de cette pratique.

La fumure minérale semble avoir des effets négatifs sur le niveau des caractéristiques du sol par rapport au témoin. Cette baisse est plus nette et significative pour le $\mathrm{pH}$ eau et les teneurs en potassium total quel que soit le mode de gestion des résidus de bananier. Concernant le $\mathrm{pH}$ eau, toutes les options apportant des engrais ont montré des valeurs inférieures à celles du témoin sans engrais. Selon Vanlauwe et Giller (2006), l'effet à court des engrais sur la baisse du $\mathrm{pH}$ du sol pourrait être attribué à la présence dans la fumure de l'urée dont la minéralisation s'accompagnerait de la production d'ions $\mathrm{H}+$ qui acidifient le sol. L'effet positif de la fumure minérale dans l'accroissement de la minéralisation de la matière organique conduisant à la libération des éléments minéraux qui seraient par la suite perdus par lixiviation et/ou par absorption des plantes, pourrait aussi justifier le fait que le $\mathrm{pH}$ soit systématique plus élevé dans le témoin par rapport autres options de fumure minérale. Pour le potassium total, la baisse des teneurs par rapport au témoin est plus importante avec les fumures ne comportant cet élément comparativement au traitement NPK.

\section{Conclusion}

L'étude menée dans l'Ouest du Burkina Faso est une contribution à la valorisation de la grande quantité de biomasse produite par la culture du bananier en rotation avec le maïs. Elle avait pour objectif d'évaluer les effets à court terme de différents modes de gestion des résidus du bananier en combinaison avec des options de fumure minérale sur les rendements du maïs et les caractéristiques chimiques du sol. Des quatre modes de gestion des résidus de bananier testés, le paillage à la surface du sol et l'enfouissement dans le sol ont amélioré les rendements du maïs par rapport au brûlage et surtout au ramassage. L'effet du paillage est apparu cependant moins marqué en culture irriguée. L'application de la fumure minérale en combinaison avec les modes de gestion des résidus de bananier a permis une augmentation significative des rendements du maïs par rapport au témoin sans engrais. Toutefois, les niveaux de rendement du maïs obtenus font ressortir deux options de fumure minérale en fonction des modes de gestion des résidus. En effet, lorsque les résidus sont paillés, enfouis ou brûlés, l'apport de l'azote seul a enregistré des rendements comparables aux autres fumures (l'azote et le phosphore; les trois éléments NPK). Par contre, en cas de ramassage des résidus, seule l'option apportant $\mathrm{N}$, $\mathrm{P}$ et $\mathrm{K}$ a permis d'accroitre significativement le rendement. Concernant les caractéristiques chimiques du sol, seul le 
ramassage des résidus a montré une baisse du niveau du $\mathrm{pH}$ et des teneurs en carbone total, azote total et en potassium disponible comparativement aux autres modes de gestion. Cependant, quel que soit le mode de gestion des résidus, l'application combinée des engrais a eu un effet dépressif plus marqué sur le niveau du $\mathrm{pH}$ et les teneurs en potassium. L'étude a montré l'intérêt du recyclage des résidus de bananier par paillage et enfouissement combiné avec la fumure azotée pour améliorer les rendements de maïs. Toutefois, la gestion durable de ces nouveaux agrosystèmes devrait intégrer les cultures de l'ensemble de la rotation.

\section{CONFLITS D'INTERETS}

Les auteurs déclarent qu'il n'existe pas de conflit d'intérêts sur cet article.

\section{CONTRIBUTIONS DES AUTEURS}

MOS et ZAM ont conduit les essais et la collecte des données de terrain sous la supervision de BB. ZAM a effectué les analyses de sols. BB et HSK ont contribué à la rédaction du manuscrit et approuvé la version finale.

\section{REMERCIEMENTS}

Les auteurs remercient vivement l'Université Nazi BONI (UNB) et International Fertilizer Development Center pour les infrastructures, les facilités administratives et leur contribution au financement de ce travail. Ils remercient également l'Institut de l'Environnement et de Recherches Agricoles (INERA) pour avoir facilité les analyses de sol.

\section{RÉFÉRENCES}

Abarchi I, Ado Aalifou H, Timbely D, ZhanYu Z, Xiang-Ping G, Wei-Mu W. 2018. Impact of drought on growth and yield of wheat as affected by mulch. Int. J. Biol. Chem. Sci., 12(4): 1824-1835. DOI: https://dx.doi.org/10.4314/ijbcs.v12i4.24

Abdou G, Ewusi-Mensah N, Nouri M, Tetteh FM, Safo EY, Abaidoo RC. 2016.
Nutrient release partterns of compost and its implication on crop yield under Sahelian conditions of Niger. Nutrient Cycling in Agroecosystems, 105(2): 117128.

DOI: http://dx.doi.org/10.1007/s10705-0169779-9

Ariste C, Kamboulé JC. 2002. La culture de la banane au Burkina Faso: manuel du producteur. Première édition. Burkina Faso. p. 55.

Autfray P, Sissoko F, Falconnier G, Ba A, Dugué P, 2012. Usages des résidus de récolte et gestion intégrée de la fertilité des sols dans les systèmes de polyculture élevage : étude de cas au Mali-Sud. Cah Agric, 21: 225-34. DOI: 10.1684/agr.2012.0568

Bacyé B, Kambiré SH, Somé SA. 2019. Effets des pratiques paysannes de fertilisation sur les caractéristiques chimiques d'un sol ferrugineux tropical lessivé en zone cotonnière à l'Ouest du Burkina Faso. Int. J. Biol. Chem. Sci., 13(6): 2930-2941. DOI:

https://dx.doi.org/10.4314/ijbcs.v13i6.39

Badou A, Akondé PT, Adjanohoun A, Adjé IT, Aïhou K et Igué AM. 2013 Effets de différents modes de gestion des résidus de soja sur le rendement du maïs dans deux zones agroécologiques du Centre-Bénin. Bulletin de la Recherche Agronomique du Bénin (BRAB), Numéro spécial, Fertilité du maïs: 34-38. http://www.slire.net/download/1800/bad ou_et_al..pdf

Barbier B, Ouedraogo H, Dembele Y, Yacouba H, Barry B, Jamin J-Y. 2011. L'agriculture irriguée dans le Sahel ouestafricain. Diversité des pratiques et des performances. Cah. Agri., 20: 24-33. DOI: 10.1684/agr.2011.0475

Barthès BG, Penche A, Hien Ed, Deleporte Ph, Clermont-Dauphin C, Cournac L, Manlay RJ. 2015. Effect of ramial wood amendment on sorghum production and topsoil quality in a Sudano-Sahelian 
ecosystem (central Burkina Faso). Agroforestry Systems, 89: 81-93. DOI: http://dx.doi.org/10.1007/s10457-0149759-5

Cattan P, Cabidoche Y-M, Lacas J-G, Voltz M. 2006. Effects of tillage and mulching on runoff under banana (Musa spp.) on a tropical Andosol. Soil and Tillage Research, 86(1): 38-51. DOI: https://doi.org/10.1016/j.still.2005.02.00 2

Dembélé Y, Zangré A. 2014. Développement de l'irrigation au Burkina Faso : Etat des lieux et caractéristiques des aménagements hydro-agricoles. Paper 6 in Irrigation in West Africa: Current Status and a View to the Future. Regassa E. Namara and Hilmy Sally, editors. IWMI Publication. Proceedings of the Workshop held in Ouagadougou, Burkina Faso, December 1-2, 2010, pp 89-110. doi:10.5337/2014.218

Djenontin JA, Amidou M, Baco NM, Wennink B. 2003. Valorisation des résidus de récolte dans l'exploitation agricole au nord du Bénin : Production de fumier et enfouissement des résidus de récolte pour la gestion de la fertilité des sols. In: Organisation spatiale et gestion des ressources et des territoires ruraux. Actes du colloque international, 25-27 février 2003, Umr Sagert, Cnearc, Montpellier, France. Dugué P, Jouve Ph, (éds.), DOI: http://10.13140/2.1.1683.8407

Fontes J, Guinko S. 1995. Carte de la végétation et de l'occupation $\mathrm{du}$ sol du Burkina Faso: Notice explicative. Université de Ouagadougou, Ouagadougou, Burkina Faso. p. 67.

Guto SN, Pypers P, Vanlauwe B, de Ridder N, Giller KE. 2012. Socio-écological niches for minimum tillage and crop-residue retention in continuous maize cropping systems in smallholder farms of Central Kenya. Agronomy Journal, 104(1): 188198. DOI: $10.2134 /$ agronj2010.0359
Kimani SK, Esilaba AO, Odera MM, Kimenye L, Vanlauwe B, Bationo A. 2007. Effects of organic and mineral sources of nutrients on maize yields in three districts of central Kenya. In Advances in Integrated Soil Fertility Management in Sub-Saharan Africa: Challenges and Opportunities, Bationo A (ed). Springer; 353-357.

https://link.springer.com/chapter/10.100 7/978-1-4020-5760-1_32

Koulibaly B, Traoré O, Dakuo D, Zombré PN, Bondé D. 2010a. Effets de la gestion des résidus de récolte sur les rendements et les bilans culturaux d'une rotation cotonnier-maïs-sorgho au Burkina Faso. TROPICULTURA, 28(3): 184-189.

Koulibaly B, Traoré O, Dakuo D, Zombré PN, Bondé D. 2010b. Effets de la valorisation des résidus de récolte sur la nutrition minérale du cotonnier et les rendements d'une rotation coton-maïs-sorgho dans l'Ouest du Burkina Faso. Int. J. Biol. Chem. Sci., 4(6): 2120-2132.

Lekasi JK, Woomer PL, Tenywa JS, Bekunda MA. 2001. Effect of mulching cabbage with banana residues on cabbage yield, soil nutrient and moisture supply, soil biota and weed biomass. African Crop Science Journal, 9(3): 499-506. DOI : http://dx.doi.org/10.4314/acsj.v9i3.2759 6.

Mason SC, Ouattara K, Taonda SJB, Palé S, Sohoro A, Kaboré D. 2014. Soil and cropping system research in semiarid West Africa as related to the potential for conservation agriculture. International Journal of Agricultural Sustainability, $13(2)$.

DOI: http://dx.doi.org/10.1080/14735903.2014 .945319

Ouédraogo M, Dembélé Y, Somé L. 2010. Perception et stratégies d'adaptation aux changements des précipitations : cas des paysans du Burkina Faso. Sécheresse, 21(2): $\quad 87-96 . \quad$ DOI: $10.1684 / \mathrm{sec} .2010 .0244$ 
Raphael L, Sierra J, Recous S, OzierLafontaine H, Desfontaines L. 2012. Soil turnover of crop residues from the banana (Musa AAA cv. Petite-Naine) mother plant and simultaneous uptake by the daughter plant of released nitrogen. European Journal of Agronomy, 36: 117 123.

DOI: https://doi.org/10.1016/j.eja.2011.07.005

Roose E. 2017. Potentiel du paillage pour réduire l'érosion et restaurer la productivité des sols tropicaux : une revue en Afrique francophone. In Restauration de la Productivité des Sols Tropicaux et Méditerranéens. Contribution à l'Agroécologie. IRD Edition; 191-199. DOI: 10.1684/agr.2010.0412

Sarr B, Kafando L, Atta S. 2011. Identification des risques climatiques de la culture du maïs au Burkina Faso. Int. J. Biol. Chem.
Sci. $\quad$ 5(4): $\quad$ 1659-1675. DOI: http://dx.doi.org/10.4314/ijbcs.v5i4.28

Sohou LR, Sintondji OL, Sogbedji MJ, Agbossou KE. 2017. Effets des résidus de récolte du riz et de l'azote sur la production du piment au Benin. Journal de la Recherche Scientifique de l'Université de Lomé, 19(2): 89-106. https://www.ajol.info/index.php/jrsul/arti cle/view/164262.

Thieuleux L. 2006. Biodisponibilité de l'azote en cultures bananières sur nitisol: application à la gestion de la fertilisation azotée. Thèse de Doctorat, Université des Antilles et de la Guyane, Antilles, p. 105. Vanlauwe B, Giller KE. 2006. Popular myths around soil fertility management in subSaharan Africa. Agriculture, Ecosystems and Environment, 116: 34-46. DOI: http://dx.doi.org/10.1016/j.agee.2006.03. 016. 